\title{
Dynamical Behaviors of a Fractional-Order Three Dimensional Prey-Predator Model
}

\author{
Noor S. Sh. Barhoom and Sadiq Al-Nassir (ic \\ Department of Mathematics, College of Science, University of Baghdad, Baghdad, Iraq \\ Correspondence should be addressed to Sadiq Al-Nassir; sadiq.n@sc.uobaghdad.edu.iq
}

Received 14 September 2021; Revised 21 October 2021; Accepted 27 November 2021; Published 15 December 2021

Academic Editor: Jaume Giné

Copyright (c) 2021 Noor S. Sh. Barhoom and Sadiq Al-Nassir. This is an open access article distributed under the Creative Commons Attribution License, which permits unrestricted use, distribution, and reproduction in any medium, provided the original work is properly cited.

\begin{abstract}
In this paper, the dynamical behavior of a three-dimensional fractional-order prey-predator model is investigated with Holling type III functional response and constant rate harvesting. It is assumed that the middle predator species consumes only the prey species, and the top predator species consumes only the middle predator species. We also prove the boundedness, the non-negativity, the uniqueness, and the existence of the solutions of the proposed model. Then, all possible equilibria are determined, and the dynamical behaviors of the proposed model around the equilibrium points are investigated. Finally, numerical simulations results are presented to confirm the theoretical results and to give a better understanding of the dynamics of our proposed model.
\end{abstract}

\section{Introduction}

The most influential theme in ecology and mathematical modeling is the dynamic of the relationship among species. Many authors extended or modified the work of the Lotka and Voltera model $[1,2]$, and they have investigated thse topics widely by using ordinary differential equations or deference equations, see [3-9], and references therein. Nowadays, authors formulate their mathematical models by fractional order differential equation due to their ability to give the precise description for various linear and nonlinear phenomena [10-15]. The increasing of mathematical models that based on fractional order differential equation has recently obtained popularity in the studying the behavior of biological models. Fractional-order differential equation has been successfully used and applied to model many areas of science, engineering, and phenomena that cannot be formulated by other types of equations $[10,16]$. In $[12,16-21]$, authors have investigated the effects of the fractional order differential equation on a prey predator model as well as they also discussed the stability analysis of equilibrium points of fractional order model with and without harvest- ing, as well as the existence, uniqueness, and boundedness of the solutions that are proved.

There are several different types of definitions of fractional-order differential equation in the literatures [16, 22-25], for e.g., Caputo, Riemann-Litouville, Fabrizio, Marechand,Grunwald-Letnikov, wayl, and Riesz fractional-order derivatives. Throughout this work, we used the Caputo fractional-order derivatives because it is not necessary to define the initial conditions of fractional-order and its fractional-order derivative of constant function is zero, as well as the similarity of the initial conditions of fractional order differential and the integer order ones [8]. This work is organized as follows: in Section 2, some useful definitions and concept that concern to the fractional order are presented. In Section 3, a three-dimensional fractional-order prey-predator model is considered. The uniqueness and boundedness as well as the nonnegativity of its solution are proved. In Section 4, all the equilibrium points are determined, and the conditions to achieve its local stability are set. In Section 5, numerical simulations are given to confirm the theoretical results. Finally, conclusions are given in Section 6. 


\section{Preliminaries}

Definition 1. [24, 26]: Caputo's definition of fractional derivatives is given as follows:

$D_{t}^{\alpha} f(t)=I^{[\alpha]-\alpha} f^{[\alpha]}(t), \alpha>0$,

where $[\alpha]$ is the least integer which is not less than $\alpha$, and $I^{\theta}$ is the Riemann-Liouville integral operator of order $\theta$ which is given by

$I^{\theta} f(t)=1 / \Gamma(\theta) \int_{0}^{t}(t-\tau)^{\theta-1} f(\tau) d \tau, \theta>0$.

$\Gamma(\theta)$ is the Euler Gamma function.

Throughout this work, we need some useful results for the fractional-order derivative. We start with the following results which are proved in $[13,16,27]$.

Lemma 1.

(1) Suppose that $f(t) \in C[a, b]$ and $D_{a}^{\alpha} f(t) \in C(a, b]$ with $0<\alpha \leq 1$, then we have

$$
f(t)=f(a)+\frac{1}{\Gamma(\alpha)}\left(D_{a}^{\alpha} f\right)(\xi)(t-a)^{\alpha}
$$

where $a \leq \xi \leq x, \forall x \in(a, b]$.

(2) Suppose $f(t) \in C[a, b]$ and $D_{t}^{\alpha} f(t) \in C(a, b), 0<\alpha \leq$ 1. If $D_{a}^{\alpha} f(t) \geq 0, \forall t \in(a, b)$, then $f(t)$ is a nondecreasing function $\forall t \in[a, b]$, and if ${ }_{t_{0}}^{c} D_{a}^{\alpha} f(t) \leq 0, \forall t \in(a, b)$ , then $f(t)$ is a nonincreasing function $\forall t \in[a, b]$

(3) Consider the Cauchy problem

$$
\begin{gathered}
D_{a}^{\alpha} x(t)=\lambda x(t)+f(t), \\
x(a)=b \quad(b \in \mathbb{R}) .
\end{gathered}
$$

With $0<\alpha \leq 1$ and $\lambda \in \mathbb{R}$, then the form of the solution is given by

$$
x(t)=b E_{\alpha}\left[\lambda(t-a)^{\alpha}\right]+\int_{a}^{t}(t-s)^{\alpha-1} E_{\alpha, \alpha}\left[\lambda(t-s)^{\alpha}\right] f(s) d s .
$$

While the solution to the problem

$$
\begin{gathered}
D_{a}^{\alpha} x(t)=\lambda x(t), \\
x(a)=b \quad(b \in \mathbb{R}),
\end{gathered}
$$

is given by

$$
x(t)=b E_{\alpha}\left[\lambda(t-a)^{\alpha}\right] .
$$

(4) Let $u(t)$ be a continuous function on $\left[t_{0}, \infty\right]$ and satisfy

$$
D_{a}^{\alpha} u(t) \leq-\lambda u(t)+\mu,
$$

where $0<\alpha<1,(\lambda, \mu) \in \mathbb{R}^{2}, \lambda \neq 0$, and $t_{0} \geq 0$ is the initial time. Then, its solution has the form

$$
\mathrm{u}(\mathrm{t}) \leq\left(\mathrm{u}_{\mathrm{t}_{0}}-\frac{\mu}{\lambda}\right) \mathrm{E}_{\alpha}\left[-\lambda\left(\mathrm{t}-\mathrm{t}_{0}\right)^{\alpha}\right]+\frac{\mu}{\lambda} .
$$

For the existence a unique solution for the general fractional-order derivative, we have the next lemma that appeared in [28].

Lemma 2. Consider the system

$D_{t}^{\alpha} x(t)=f(t, x), t>t_{0}$

with the initial condition $x_{t_{0}}$, where $0<\alpha \leq 1, f:\left[t_{0}, \infty\right)$ $\times \Omega \longrightarrow \mathbb{R}^{n}$. If $f(t, x)$ satisfies the locally Lipchitz condition with respect to $x$, then there exists a unique solution of the above system on $\left[t_{0}, \infty\right) \times \Omega$.

\section{Model Formulation}

Frist, the following three dimension prey-predator model with functional response of Holling type III is considered as follows:

$$
\left.\begin{array}{l}
\frac{d X}{d T}=R_{0} X\left(1-\frac{X}{K_{0}}\right)-\frac{a_{1} Y X^{2}}{B_{1}+X^{2}} \\
\frac{d Y}{d T}=\frac{a_{2} Y X^{2}}{B_{1}+X^{2}}-\frac{b_{1} Z Y^{2}}{B_{2}+Y^{2}}-c_{1} Y-h_{2} Y \\
\frac{d Z}{d T}=\frac{b_{2} Z Y^{2}}{B_{2}+Y^{2}}-c_{2} Z
\end{array}\right\},
$$

where $X, Y$, and $Z$ are the density of prey species, middle species, and the top predator species. $R_{0}, K_{0}, c_{1}, c_{2}$, and $b_{1}$ are the intrinsic growth rates, the carrying capacity of the prey population, the death rates for middle predator population and top predator population, and capture rate of prey and middle predator, respectively. The parameter $a_{1}$ is the conservation rate of prey $X$, and the parameters $a_{2}$ and $b_{2}$ are the conservation rate of middle predator $Y$ to the top predator $Z$, respectively. The constants $B_{1}$ and $B_{2}$ are the predation rates and half saturation constants, respectively. The parameter $h_{2}$ denotes to the constant harvesting rate.

We use the following transformation to reduce the dimension of model (8). $x=X / k, y=\left(a_{1} / k_{0} R_{0}\right) Y$ , $z=\left(a_{1} b_{1} / R_{0}^{2} k_{0}\right) Z$, and $t=R_{0} T$, and then we obtain the following system:

$$
\left.\begin{array}{l}
\frac{d x}{d t}=x(1-x)-\frac{a x^{2} y}{1+a x^{2}} \\
\frac{d y}{d t}=\frac{b x^{2} y}{1+a x^{2}}-\frac{d y^{2} z}{1+d y^{2}}-m_{1} y-h y \\
\frac{d z}{d t}=\frac{c y^{2} z}{1+d y^{2}}-m_{2} z
\end{array}\right\},
$$


where $a=k_{0}^{2} / B_{1}, \quad b=a a_{2} / R_{0}, c=R_{0} d / b_{2}, d=k_{0}^{2} R_{0}^{2} / a_{1}^{2} B_{2}$, $m_{1}=c_{1} k_{0}^{2} R_{0} / a_{1}, m_{2}=R_{0} / c_{2}$, and $h=h_{2} k_{0} R_{0} / a_{1}$.

Next, we introduce the fractional-order derivative $\alpha$ in model (2) with the help of fractional order Caputo type derivative. Then, the system (9) becomes as follows:

$$
\left.\begin{array}{l}
D_{t}^{\alpha} x(t)=x(1-x)-\frac{a x^{2} y}{1+a x^{2}} \\
D_{t}^{\alpha} y(t)=\frac{b x^{2} y}{1+a x^{2}}-\frac{d y^{2} z}{1+d y^{2}}-m_{1} y-h y \\
D_{t}^{\alpha} z(t)=\frac{c y^{2} z}{1+d y^{2}}-m_{2} z
\end{array}\right\}
$$

Remark 3. Because of the biological significance, we are interesting solutions that are nonnegative and bounded only, so that we will prove the following theorem.

Theorem 4. Let $\Omega_{+}=\{(x, y, z) \mid x \geq 0, y \geq 0$ and $z \geq 0\}$ and denotes all nonnegative real numbers in $\mathbb{R}^{3}$, and then all solutions of the system (10) with $x_{0} \geq 0, y_{0} \geq 0$ and $z_{0} \geq 0$ are uniformly bounded and nonnegative.

Proof. we have to show that $x(t) \geq 0, \forall t \geq 0$, assuming $x(0)$ $>0$ for $t=0$ considering that $x(t) \geq 0, \forall t \geq 0$ is not true. Then, there exists a constant $t_{1}>0$ such that

$$
\begin{cases}x(t)>0, & 0 \leq t<t_{1}, \\ x(t)=0, & t=t_{1}, \\ x(t)<0, & t>t_{1} .\end{cases}
$$

From the first equation of system (10), we have

$$
\left.D_{t_{1}}^{\alpha} x(t)\right|_{t=t_{1}}=0
$$

According to part 1 in Lemma 1 , we have $x\left(t_{1}^{+}\right)=0$, which contradicts the fact that $x\left(t_{1}^{+}\right)<0$, i.e., $x(t)<0, t>t_{1}$. Therefore, we have $x(t) \geq 0, \forall t \geq 0$. By the same arguments, $y(t) \geq 0, \forall t \geq 0$ and $z(t) \geq 0$ can get $\forall t \geq 0$. For the uniformly bounded, Let $V(t)=(1 / a) x+(1 / b) y+(d / b c) z$, and then we have

$$
\begin{aligned}
D^{\alpha} V(t) & =\frac{1}{a} D^{\alpha} x(t)+\frac{1}{b} D^{\alpha} y(t)+\frac{d}{b c} D^{\alpha} z(t) \\
& =\frac{1}{a} x(1-x)-\frac{1}{b}\left(m_{1}+h\right) y-\frac{d}{b c} m_{2} z
\end{aligned}
$$

Now, for each $\eta>0$, we have

$$
\begin{aligned}
D^{\alpha} V(t)+\eta V(t)= & \frac{1}{a} x(1-x)-\frac{1}{b}\left(m_{1}+h\right) y-\frac{d}{b c} m_{2} z+\eta \frac{1}{a} x \\
& +\eta \frac{1}{b} y+\eta \frac{d}{b c} z \leq-\frac{1}{a} x^{2}+(\eta+1) \frac{1}{a} x \\
& +\left(\eta-\left(m_{1}+h\right)\right) \frac{1}{b} y+\left(\eta-m_{2}\right) \frac{d}{b c} z \\
\leq & -\frac{1}{a}\left[x^{2}-(\eta+1) x+\frac{1}{4}(\eta+1)^{2}-\frac{1}{4}(\eta+1)^{2}\right] \\
& +\left(\eta-\left(m_{1}+h\right)\right) \frac{1}{b} y+\left(\eta-m_{2}\right) \frac{d}{b c} z \\
= & -\frac{1}{a}\left(x-\frac{1}{2}(\eta+1)\right)^{2}+\frac{(\eta+1)^{2}}{4 a} \\
& +\left(\eta-\left(m_{1}+h\right) \frac{1}{b} y+\left(\eta-m_{2}\right) \frac{d}{b c} z\right. \\
\leq & \frac{1}{4 a}(\eta+1)^{2}+\left(\eta-\left(m_{1}+h\right)\right) \frac{1}{b} y+\left(\eta-m_{2}\right) \frac{d}{b c} z .
\end{aligned}
$$

Therefore,

$D^{\alpha} V(t)+\eta V(t) \leq l$, by taking $\eta<\min \left(\left(m_{1}+h\right), m_{2}\right)$, where $l=(1 / 4 a)(\eta+1)^{2}>0$.

Now by using part 4 in Lemma 1, we have

$$
\begin{aligned}
\mathrm{V}(t) & \leq\left(V(0)-\frac{l}{\eta}\right) E_{\alpha}\left[-\eta(t-0)^{\alpha}\right]+\frac{l}{\eta}, \\
& \leq V(0) E_{\alpha}\left[-\eta t^{\alpha}\right]+\frac{l}{\eta}\left(1-E_{\alpha}\left[-\eta t^{\alpha}\right]\right) .
\end{aligned}
$$

Thus, $\quad V(t) \longrightarrow l / \eta$ as $t \longrightarrow \infty$ and $0<V(t) \leq l / \eta$. Therefore, all solutions of system (10) that starts from $\Omega_{+}$are confined in the region $\Omega=\left\{(x, y, z) \in \mathbb{R}^{3} \mid V(t) \leq\right.$ $(l / \eta)+\varepsilon$, for any $\varepsilon>0\}$.

Now, the existence and uniqueness of the solution of the considered system (10) are shown in the next theorem:

Theorem 5. Let $\gamma$ be a sufficiently large, and then for each $S_{0}=\left(x_{0}, y_{0}, z_{0}\right) \in\left\{(x, y, z) \in \mathbb{R}^{3} \mid \max \{|x|,|y|,|z| \leq \gamma\}\right\}$,

there exists a unique solution $S=(x, y, z) \in \Omega$ of the fractional-order system (10) with initial condition $S_{0}$, which is defined for all $t \geq 0$.

Proof. Let $S=(x, y, z), \bar{S}=(\bar{x}, \bar{y}, \bar{z})$, and consider a mapping $H(S)=\left(H_{1}(S), H_{2}(S), H_{3}(S)\right)$, such that

$$
\left.\begin{array}{l}
H_{1}(S)=x(1-x)-\frac{a x^{2} y}{1+a x^{2}} \\
H_{2}(S)=\frac{b x^{2} y}{1+a x^{2}}-\frac{d y^{2} z}{1+d y^{2}}-m_{1} y-h y \\
H_{3}(S)=\frac{c y^{2} z}{1+d y^{2}}-m_{2} z
\end{array}\right\} .
$$


For any $S, \bar{S} \in \Omega$, we have

$$
\begin{aligned}
\|H(S)-H(\bar{S})\|= & \left|H_{1}(S)-H_{1}(\bar{S})\right|+\left|H_{2}(S)-H_{2}(\bar{S})\right| \\
& +\left|H_{3}(S)-H_{3}(\bar{S})\right|=\mid x(1-x)-\frac{a x^{2} y}{1+a x^{2}} \\
& -\bar{x}(1-\bar{x})+\frac{a \bar{x}^{2} \bar{y}}{1+a \bar{x}^{2}}|+| \frac{b x^{2} y}{1+a x^{2}}-\frac{d y^{2} z}{1+d y^{2}} \\
& -m_{1} y-h y-\frac{b \bar{x}^{2} \bar{y}}{1+a \bar{x}^{2}}+\frac{d \bar{y}^{2} \bar{z}}{1+d \bar{y}^{2}}+m_{1} \bar{y}+h \bar{y} \mid \\
& +\left|\frac{c \bar{y}^{2} \bar{z}}{1+d \bar{y}^{2}}-m_{2} z-\frac{c \bar{y}^{2} \bar{z}}{1+d \bar{y}^{2}}+m_{2} \bar{z}\right| \\
= & \mid(x-\bar{x})(1-(x+\bar{x})) \\
& -a\left(\frac{\left(x^{2} y-\bar{x}^{2} \bar{y}\right)+a x^{2} \bar{x}^{2}(y-\bar{y})}{\left(1+a x^{2}\right)\left(1+a \bar{x}^{2}\right)}\right) \mid \\
& +\mid b\left(\frac{\left(x^{2} y-\bar{x}^{2} \bar{y}\right)+a x^{2} \bar{x}^{2}(y-\bar{y})}{\left(1+a x^{2}\right)\left(1+a \bar{x}^{2}\right)}\right) \\
& -d\left(\frac{\left.\left(y^{2} z-\bar{y}^{2} \bar{z}\right)+d y^{2} \bar{y}^{2}(z-\bar{z})\right)}{\left(1+d y^{2}\right)\left(1+d \bar{y}^{2}\right)}\right) \\
& -m_{1}(y-\bar{y})-h(y-\bar{y}) \mid \\
& +\left|c\left(\frac{\left.\left(y^{2} z-\bar{y}^{2} \bar{z}\right)+d y^{2} \bar{y}^{2}(z-\bar{z})\right)}{\left(1+d y^{2}\right)\left(1+d \bar{y}^{2}\right)}\right)-m_{2}(z-\bar{z})\right| .
\end{aligned}
$$

Since $\left|1 /\left(1+d y^{2}\right)\left(1+d \bar{y}^{2}\right)\right| \leq 1$ and $\mid 1 /\left(1+a x^{2}\right)(1+a$ $\left.\bar{x}^{2}\right) \mid \leq 1$ and $\max \{|x|,|y|,|z|\} \leq \gamma$, we can have

$$
\begin{aligned}
\|H(S)-H(\bar{S})\| \leq & |x-\bar{x}||1-(x+\bar{x})| \\
& +(a+b) \frac{\left|x^{2} y-\bar{x}^{2} \bar{y}\right|+a\left|x^{2}\right|\left|\bar{x}^{2}\right||y-\bar{y}|}{\left|\left(1+a x^{2}\right)\left(1+a \bar{x}^{2}\right)\right|} \\
& +(d+c) \frac{\left|y^{2} z-\bar{y}^{2} \bar{z}\right|+d\left|y^{2}\right|\left|\bar{y}^{2}\right||z-\bar{z}|}{\left|\left(1+d y^{2}\right)\left(1+d \bar{y}^{2}\right)\right|} \\
& +\left(m_{1}+h\right)|y-\bar{y}|+m_{2}|z-\bar{z}| \\
\leq & \left(1+2 \gamma+2 \gamma^{2}(a+b)\right)|x-\bar{x}| \\
& +\left((a+b) \gamma^{2}+a(a+b) \gamma^{4}+2 \gamma^{2}(d+c)\right. \\
& \left.+\left(m_{1}+h\right)\right)|y-\bar{y}|+\left((d+c) \gamma^{2}\right. \\
& \left.+d(d+c) \gamma^{4}+m_{2}\right)|z-\bar{z}|=L\|S-\bar{S}\|,
\end{aligned}
$$

where $L=\max \left\{\left(1+2 \gamma+2 \gamma^{2}(a+b)\right),\left((a+b) \gamma^{2}+a(a\right.\right.$ $\left.+b) \gamma^{4}+2 \gamma^{2}(d+c)+\left(m_{1}+h\right)\right),\left((d+c) \gamma^{2}+d(d+c) \gamma^{4}+\right.$ $\left.\left.m_{2}\right)\right\}$.

Therefore, $H(S)$ satisfies the Lipchitz condition. By Lemma 2, the proof is finished.

\section{The Equilibrium Points and Local Stability Analysis}

In this section, the existence and the local stability of equilibrium points of the considered system (10) are studied and investigated. Before that, we need the following theorem.
Theorem 6 (see [29]). Consider the fractional-order differential system $d^{\alpha} x / d t^{\alpha}=f(x) ; x(0)=x_{0}$, with $\alpha \in(0,1)$ and $x \in$ $\mathbb{R}^{n}$. The equilibrium points of the above system are solutions to the equation $f(x)=0$. An equilibrium point is locally asymptotically stable if all the eigenvalues $\lambda_{j}$ of the Jacobian matrix $J=\partial f / \partial x$ evaluated at the equilibrium satisfy |arg $\left(\lambda_{j}\right) \mid>\alpha \pi / 2$.

Proof (see [29]). To obtain the equilibrium points of the considered system (10), we solve the following simultaneous equations:

$$
\begin{aligned}
& D_{t}^{\alpha} x(t)=0, \\
& D_{t}^{\alpha} y(t)=0, \\
& D_{t}^{\alpha} z(t)=0 .
\end{aligned}
$$

Thus, the considered system (10) has the following possible equilibrium points:

(1) The trivial equilibrium point $E_{0}=(0,0,0)$ and the equilibrium point $E_{1}=(1,0,0)$ without any restriction on the parameters

(2) The top predator free equilibrium point $E_{2}=(\bar{x}, \bar{y}, 0)$ exists if $b>a\left(m_{1}+h\right)$ and $0<\bar{x}<1$, where $\bar{x}=$ $\sqrt{m_{1}+h / b-a\left(m_{1}+h\right)}$ and $\bar{y}=(1 / a x)(1-\bar{x})(1+a$ $\left.\bar{x}^{2}\right)$

(3) The interior equilibrium point $E_{3}=\left(x^{*}, y^{*}, z^{*}\right)$ exists if $c>m_{3} d$ and $b x^{* 2} / 1+a x^{* 2}>\left(m_{1}+h\right)$, where $y^{*}=$ $\sqrt{m_{2} / c-m_{2} d}, x^{*}$, is the positive root of the equation $a x^{3}-a x^{2}+\left(a y^{*}+1\right) x-1=0$ and $z^{*}=\left(1+d y^{* 2} / d\right.$ $\left.y^{*}\right)\left(b x^{* 2} / 1+a x^{* 2}-\left(m_{1}+h\right)\right)$ by

The Jacobian matrix of system $(10)$ at $(x, y, z)$ is given

$$
J(x, y, z)=\left[\begin{array}{ccc}
1-2 x-\frac{2 a x y}{k^{2}} & -\frac{a x^{2}}{k} & 0 \\
\frac{2 b x y}{k^{2}} & \frac{b x^{2}}{k}-\frac{2 d y z}{k_{1}^{2}}-m_{1}-h & -\frac{d y^{2}}{k_{1}} \\
0 & \frac{2 c y z}{k_{1}^{2}} & \frac{c y^{2}}{k_{1}}-m_{2}
\end{array}\right],
$$

where $k=\left(1+a x^{2}\right)$ and $k_{1}=\left(1+d y^{2}\right)$.

The next theorems give the local stability of $E_{0}, E_{1}$, and $E_{2}$, respectively.

Theorem 7. The trivial equilibrium point $E_{0}$ of the system (10) is always unstable point. 
Proof. The Jacobian matrix $J$ at $E_{0}$ is given by

$$
J\left(E_{0}\right)=\left[\begin{array}{ccc}
1 & 0 & 0 \\
0 & -\left(m_{1}+h\right) & 0 \\
0 & 0 & -m_{2}
\end{array}\right]
$$

The characteristic equation of the Jacobian matrix $J\left(E_{0}\right)$ is given by

$$
(1-\lambda)\left(-\left(\mathrm{m}_{1}+\mathrm{h}\right)-\lambda\right)\left(-\mathrm{m}_{2}-\lambda\right)=0
$$

Therefore, the roots of the above characteristic equation are $\lambda_{1}=1, \lambda_{2}=-\left(m_{1}+h\right)$, and $\lambda_{3}=-m_{3}$. Then, $\left|\arg \left(\lambda_{1}\right)\right|$ $=0<\alpha \pi / 2,\left|\arg \left(\lambda_{2}\right)\right|=\pi>\alpha \pi / 2$ and also $\left|\arg \left(\lambda_{3}\right)\right|=\pi>\alpha$ $\pi / 2$. Hence, the trivial equilibrium point is always unstable point.

Theorem 8. The equilibrium point $E_{1}$ of system (10) is locally asymptotically stable if $<\left(m_{1}+h\right)(1+a)$.

Proof. The Jacobian matrix $J$ at $E_{1}$ is given by

$$
J\left(E_{1}\right)=\left[\begin{array}{ccc}
-1 & \frac{-a}{1+a} & 0 \\
0 & \frac{b}{(1+a)}-\left(m_{1}+h\right) & 0 \\
0 & 0 & -m_{2}
\end{array}\right] \text {. }
$$

The characteristic equation of the Jacobian matrix $J\left(E_{1}\right)$ is given by

$$
(-1-\lambda)\left(\frac{b}{(1+a)}-\left(m_{1}+h\right)-\lambda\right)\left(-m_{2}-\lambda\right)=0
$$

The roots of the above characteristic equation are $\lambda_{1}=$ $-1, \lambda_{2}=b /(1+a)-\left(m_{1}+h\right)$ and $\lambda_{3}=-m_{2}$. Then, $\left|\arg \left(\lambda_{1}\right)\right|$ $=\left|\arg \left(\lambda_{2}\right)\right|=\pi>\alpha \pi / 2$ and $\left|\arg \left(\lambda_{3}\right)\right|=\pi>\alpha \pi / 2$. Hence, the equilibrium point $E_{1}$ is locally asymptotically stable if < $\left(m_{1}+h\right)(1+a)$. Otherwise, it is unstable point.

Theorem 9. The equilibrium point $E_{2}$ is locally asymptotically stable if one of the following holds:

(i) $c y^{2} / k_{1}<m_{2} l_{1}<0, l_{2}>0$, and $\Delta \geq 0$

(ii) $c y^{2} / k_{1}<m_{2} l_{1}<0$ and $\Delta<0$ and $\left|\arg \left(\lambda_{i}\right)\right|=\tan ^{-1}($ $\left.\sqrt{-\Delta} / l_{1}\right)>\alpha \pi / 2, i=2,3$ where $\lambda_{2,3}=l_{1} \mp \sqrt{l_{1}^{2}-4 l_{2}} / 2$, $l_{1}=1-2 x-\left(2(1-x)-b x^{2} / k\right)-\left(m_{1}+h\right), l_{2}=\left(b x^{2} /\right.$ k) $(1-2 x)-\left(m_{1}+h\right)(1-2 x-2(1-x) / k)$, and $\Delta=$ $l_{1}^{2}-4 l_{2}$
Proof. The Jacobian matrix $J$ at $E_{2}$ is given by

$$
J\left(E_{2}\right)=\left[\begin{array}{ccc}
1-2 x-\frac{2 a x y}{k^{2}} & -\frac{a x^{2}}{k} & 0 \\
\frac{2 b x y}{k^{2}} & \frac{b x^{2}}{k}-\left(m_{1}+h\right) & -\frac{d y^{2}}{k_{1}} \\
0 & 0 & \frac{c y^{2}}{k_{1}}-m_{2}
\end{array}\right] .
$$

The characteristic equation of the Jacobian matrix $J\left(E_{2}\right)$ is given by

$$
\left(\frac{c y^{2}}{k_{1}}-m_{2}-\lambda\right)\left(\lambda^{2}-l_{1} \lambda+l_{2}\right)=0 .
$$

It is clear that $\lambda_{1}=c y^{2} / k_{1}-m_{2}$ is negative if $c y^{2} / k_{1}<m_{2}$ so that $\left|\arg \left(\lambda_{1}\right)\right|=\pi>\alpha \pi / 2$. The other two roots $\lambda_{2}$ and $\lambda_{3}$ are $\lambda_{2,3}=l_{1} \mp \sqrt{l_{1}^{2}-4 l_{2}} / 2$. Hence, if $\Delta \geq 0$. then the eigenvalue $\lambda_{2}$ and the eigenvalue $\lambda_{3}$ are taken negative value; thus, $\arg \left(\lambda_{2}\right)|=| \arg \left(\lambda_{3}\right) \mid=\pi>\alpha \pi / 2$. Therefore, the point $E_{2}$ is locally asymptotically stable. Also, if $\Delta<0$ and if $\left|\arg \left(\lambda_{i}\right)\right|$ $=\tan ^{-1}\left(\sqrt{-\Delta} / l_{1}\right)>0, i=2,3$ hold, then the equilibrium point $E_{2}$ is asymptotically stable. Otherwise, it is unstable point.

In order to discuss the local stability of the interior point, we need the following definition and lemma.

Definition 10 (see [30]). The discriminant $D(f)$ of a polynomial

$$
f(x)=x^{n}+a_{1} x^{n-1}+a_{2} x^{n-2}+\cdots+a_{n}
$$

is defined by $D(f)=(-1)^{n(n-1) / 2} R\left(f, f^{\prime}\right)$, where $f^{\prime}$ is the derivative of $f$ and where $g(x)=x^{n}+b_{1} x^{l-1}+b_{2} x^{l-2}+\cdots+$ $b_{l}$ and $R(f, g)$ is an $(n+l) \otimes(n+l)$ determinant. If $n=3$, then $D(f)$ is given by

$$
D(f)=18 a_{1} a_{2} a_{3}+\left(a_{1} a_{2}\right)^{2}-4 a_{3}\left(a_{1}\right)^{3}-4\left(a_{2}\right)^{3}-27\left(a_{3}\right)^{2} .
$$

Lemma 11 (see [30]). Let $D(p)$ be a discriminant of the cubic polynomial equation, $p(\lambda)=\lambda^{3}+a_{1} \lambda^{2}+a_{2} \lambda+a_{3}=0$, then

(1) If $D(p)>0, a_{1}>0, a_{3}>0, a_{1} a_{2}>a_{3}$, then $\left|\arg \left(\lambda_{i}\right)\right|>$ $\alpha \pi / 2, i=1,2,3 \forall \alpha \in(0,1)$

(2) If $D(p)<0, a_{1} \geq 0, a_{2} \geq 0$, and $a_{3}>0$, then $\left|\arg \left(\lambda_{i}\right)\right|$ $>\alpha \pi / 2, i=1,2,3$ for $\alpha<2 / 3$

(3) If $D(P)<0, a_{1}<0, a_{2}<0$, and $\alpha>2 / 3$, then $\left|\arg \left(\lambda_{i}\right)\right|$ $<\alpha \pi / 2, i=1,2,3$

(4) If $D(P)<0, a_{1}>0, a_{2}>0$, and $a_{1} a_{2}=a_{3}$, then $\mid \arg$ $\left(\lambda_{i}\right) \mid>\alpha \pi / 2, i=1,2,3 . \forall \alpha \in(0,1)$ 


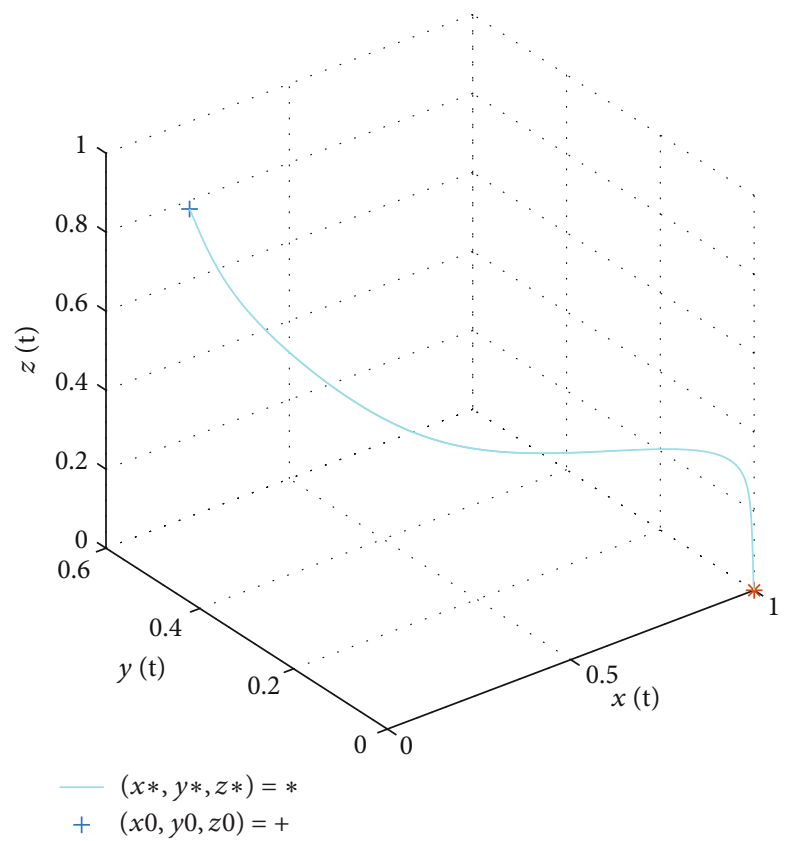

(a)

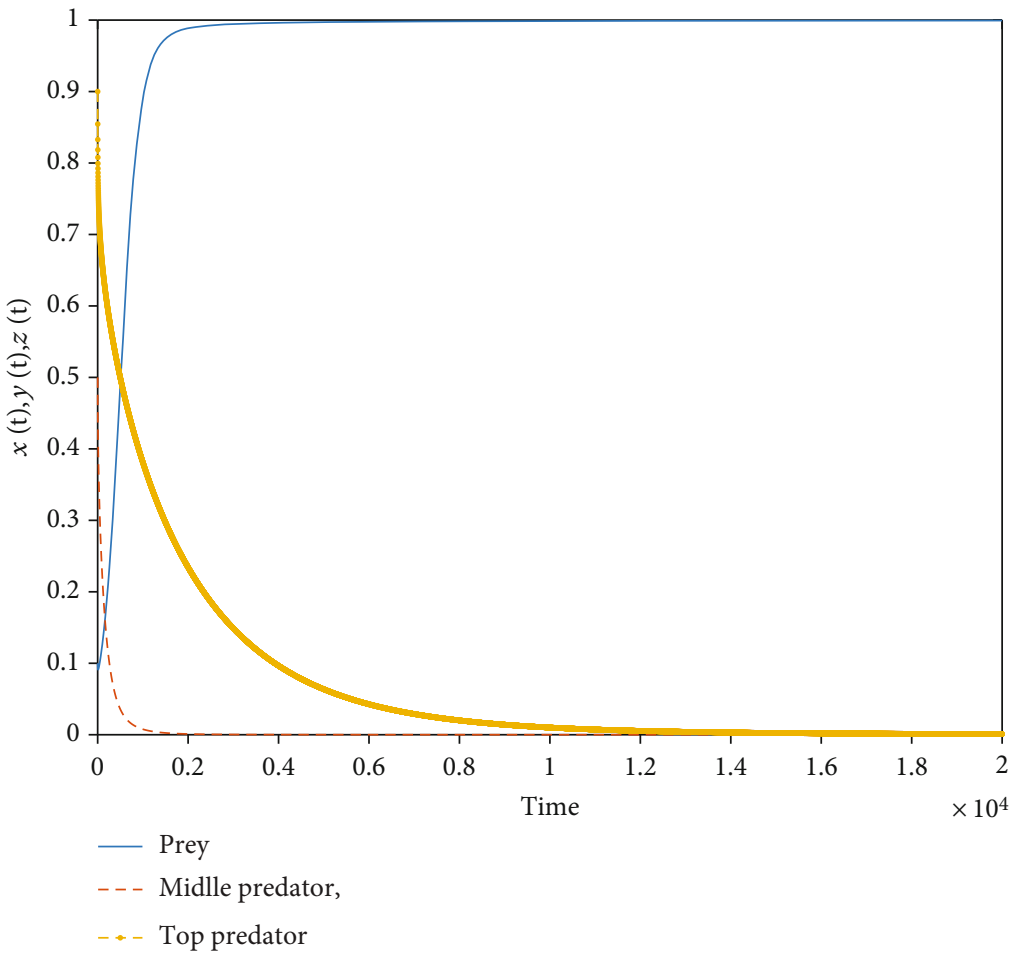

(b)

FIgURE 1: This figure shows the local stability of the point $E_{1}$ and the time series of the three species. (a) The point $E_{1}$. (b) Time series of the three species.

Proof (see [30]).

Theorem 12. The interior equilibrium point $E_{3}$ is locally asymptotically stable if one of the following conditions holds:

(1) If $D(p)>0, N_{1}>0, N_{3}>0$ and $N_{1} N_{2}-N_{3}>0$, for 0 $<\alpha<1$
(2) If $D(p)<0, N_{1} \geq 0, N_{2} \geq 0, N_{3}>0$, for $0<\alpha<2 / 3$

(3) If $D(p)<0, N_{1}>0, N_{2}>0, N_{1} N_{2}=N_{3}$, for $0<\alpha<1$

where $N_{1}=-1+2 x+\left(2 a x y / k^{2}\right)-\left(b x^{2} / k\right)+\left(2 d y z / k_{1}^{2}\right)+$ $\left(m_{1}+h\right)-\left(c y^{2} / k_{1}\right)+m_{2}, \quad N_{2}=\left(1-2 x-2 a x y / k^{2}\right)\left(b x^{2} / k-2\right.$ $\left.d y z / k_{1}^{2}-\left(m_{1}+h\right)+\left(c y^{2} / k_{1}\right)-m_{2}\right)+\left(b x^{2} / k-2 d y z / k_{1}^{2}-\left(m_{1}\right.\right.$ 


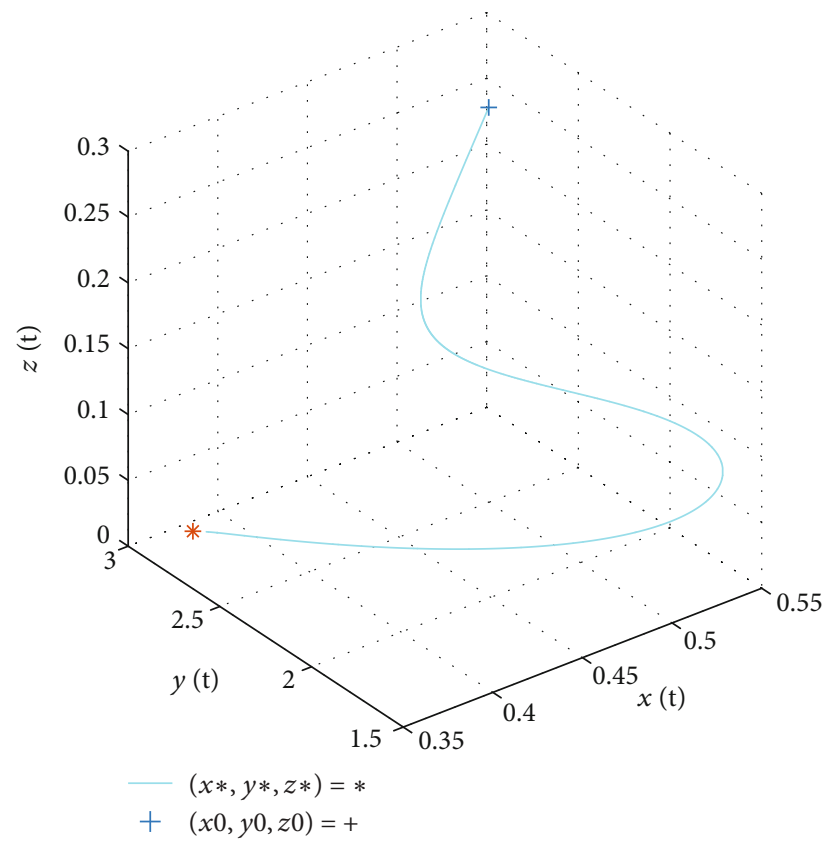

(a)

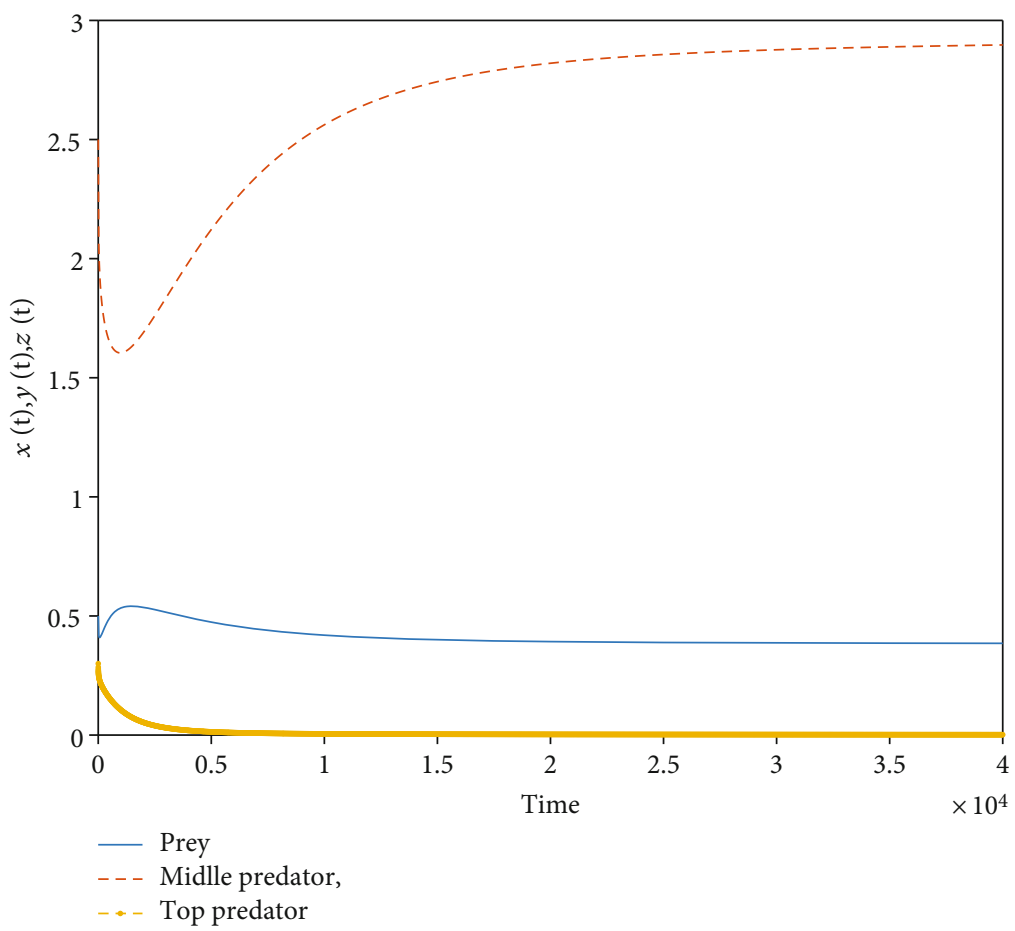

(b)

Figure 2: This figure shows the local stability of the point $E_{2}$ and the time series of the three species. (a) The point $E_{2}$. (b) Time series of the three species. 


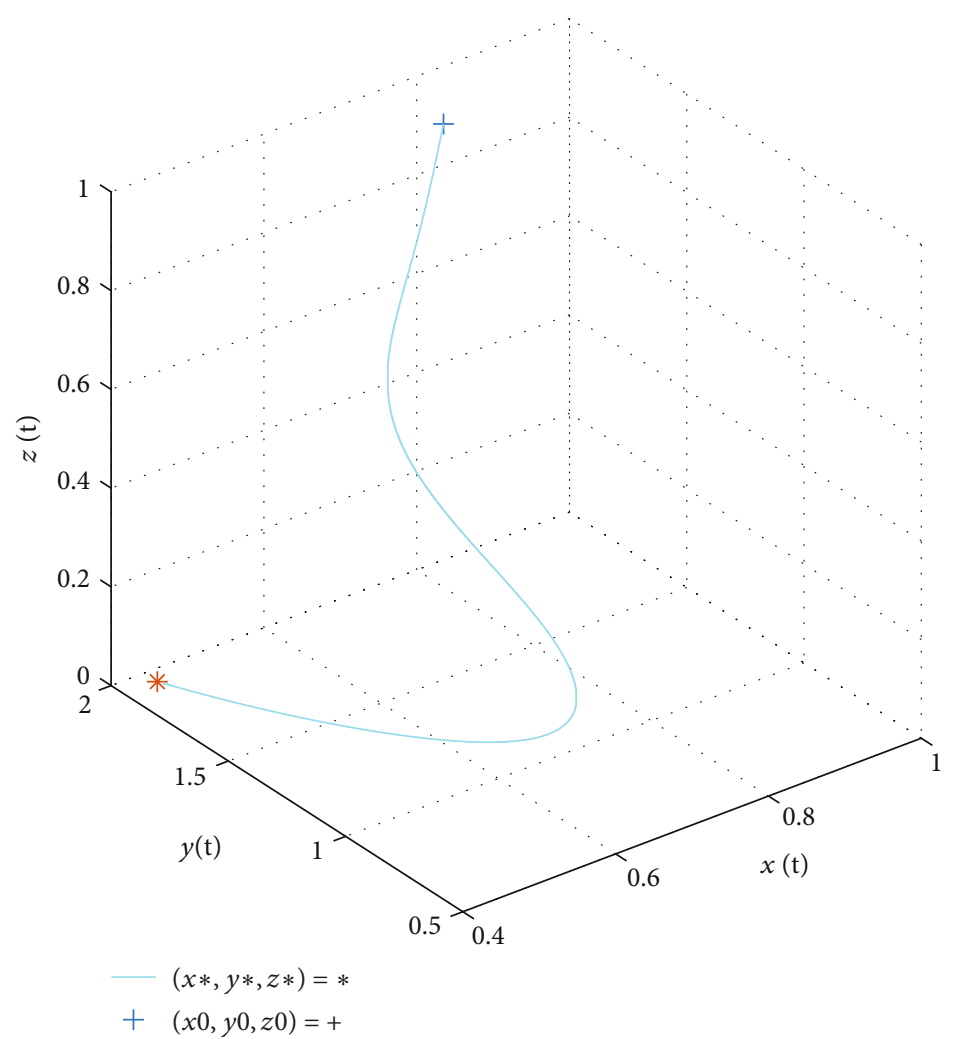

(a)

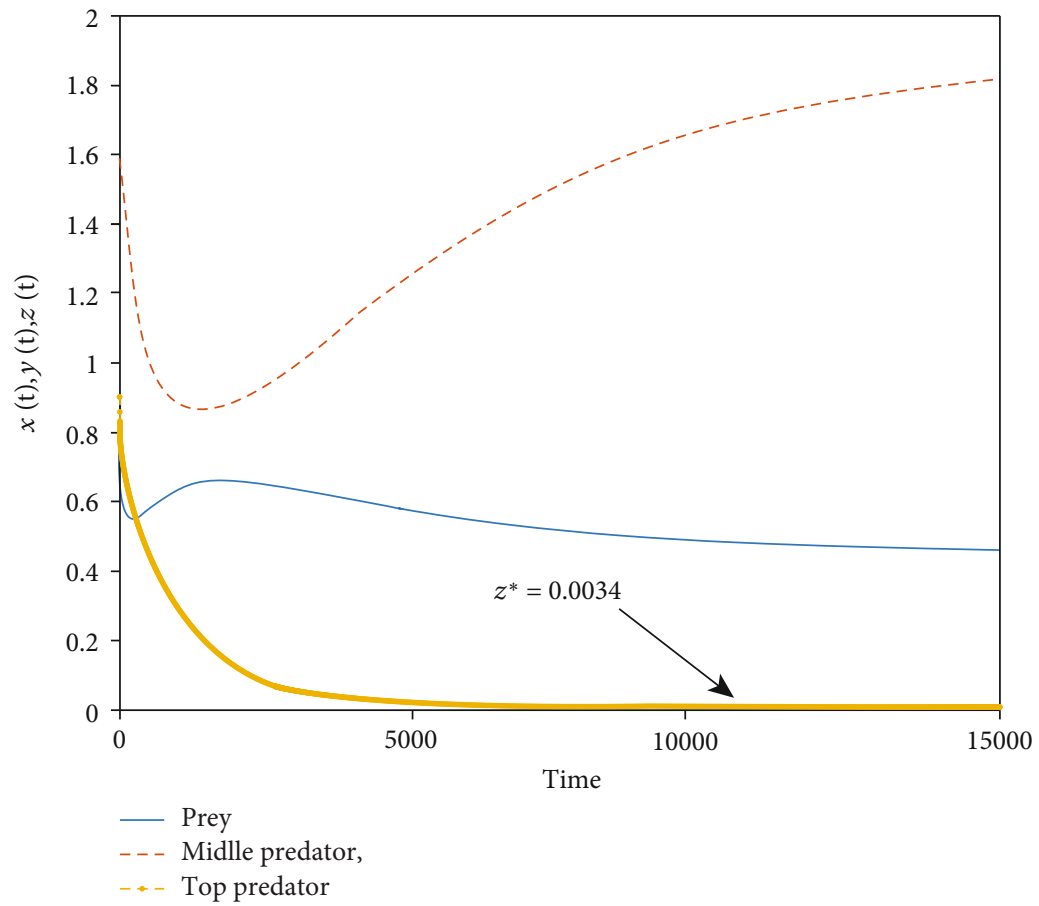

(b)

FIgURE 3: This figure shows the local stability of the point $E_{3}$ and the time series of the three species. (a) The point $E_{3}$. (b) Time series of the three species. 
$+h))\left(c y^{2} / k_{1}-m_{2}\right)+\left(2 a b x^{3} y / k^{3}\right)+\left(2 c d y^{3} z / k_{1}^{3}\right)$, and $N_{3}=$ $-\left(1-2 x-2 a x y / k^{2}\right)\left[\left(b x^{2} / k-2 d y z / k_{1}^{2}-\left(m_{1}+h\right)\right)\left(c y^{2} / k_{1}-\right.\right.$ $\left.\left.m_{2}\right)+\left(2 c d y^{3} z / k_{1}^{3}\right)\right]-\left(2 a b x^{3} y / k^{3}\right)\left(c y^{2} / k_{1}-m_{2}\right)$.

Proof. The Jacobian matrix of system (10) at $E_{3}$ is given by

$$
J\left(E_{3}\right)=\left[\begin{array}{ccc}
1-2 x-\frac{2 a x y}{k^{2}} & -\frac{a x^{2}}{k} & 0 \\
\frac{2 b x y}{k^{2}} & \frac{b x^{2}}{k}-\frac{2 d y z}{k_{1}^{2}}-\left(m_{1}+h\right) & -\frac{d y^{2}}{k_{1}} \\
0 & \frac{2 c y z}{k_{1}^{2}} & \frac{c y^{2}}{k_{1}}-m_{2}
\end{array}\right] .
$$
by

Therefore, the characteristic equation of $J\left(E_{3}\right)$ is given

$$
\mathrm{p}(\lambda)=\lambda^{3}+\mathrm{N}_{1} \lambda^{2}+\mathrm{N}_{2} \lambda+\mathrm{N}_{3}=0
$$

Thus, the discriminant $D(p)$ of the cubic polynomial $p(\lambda)$ is

$$
D(p)=-\left|\begin{array}{ccccc}
1 & N_{1} & N_{2} & N_{3} & 0 \\
0 & 1 & N_{1} & N_{2} & N_{3} \\
3 & 2 N_{1} & N_{2} & 0 & 0 \\
0 & 3 & 2 N_{1} & N_{2} & 0 \\
0 & 0 & 3 & 2 N_{1} & N_{2}
\end{array}\right|
$$
$27 N_{3}^{2}$.

where $D(p)=18 N_{1} N_{2} N_{3}+\left(N_{1} N_{2}\right)^{2}-4 N_{1}^{3} N_{3}-4 N_{2}^{3}-$

Then, all results can be obtained by Lemma 6 .

\section{Numerical Simulation}

All previous theoretical results in Section 4 are confirmed by giving numerical simulations. At different set of values of parameters, the local stability of $E_{1}, E_{2}$, and $E_{3}$ of the considered system (10) is investigated numerically.

First, for the point $E_{1}$, the following set of values of the parameters is chosen : $a_{1}=0.5 ; c_{1}=0.1 ; b_{1}=0.5 ; d_{1}=0.2$; $m_{1}=0.15 ; m_{2}=0.1 ; h=0.8, \alpha=0.98$, and $(0.1,0.5,0.9)$ is the initial value. Therefore, the condition in Theorem 4 is satisfied. Figure 1 shows that the point $E_{1}$ is locally stable.

For the point $E_{2}$, we choose the values of parameters as following $E_{2}, a_{1}=0.6 ; c_{1}=0.1 ; b_{1}=0.3 ; d_{1}=0.2 ; m_{1}=0.03$; $m_{2}=0.32$; and $h=0.01, \alpha=0.95$, and $(0.5,2.5,03)$ is the initial value. According to Theorem 9, the local stability of $E_{2}$ is satisfied. Figure 2 illustrates the local stability of the point $E_{2}$.

To show the local stability of the unique positive equilibrium point $E_{3}$, these values of parameters are chosen $a_{1}=$ $0.75 ; c_{1}=0.17 ; b_{1}=0.3 ; d_{1}=0.3 ; m_{1}=0.03 ; m_{2}=0.3 ; h=$ 0.02 , and $\alpha=0.95,(0.82,1.95,0.9)$ is the initial value so that according to Theorem 12, the equilibrium point $E_{3}$ is local stability point, and the trajectories of prey and predator are shown in Figure 3.

\section{Conclusions}

Fractional-order differential equations have recently been successfully used and applied to model many areas of science, especially, phenomena that cannot be formulated by other types of equations, so that this work concerns a study of a three-dimensional fractional-order prey-predator model with Holling type III functional response and constant rate harvesting. It is shown that the model possesses the existence, nonnegativity, boundedness, and uniqueness of the solutions, as desired in any population dynamics. The local stability of all possible equilibrium points of the considered system is investigated analytically then confirms by numerical verifications.

\section{Data Availability}

All simulations in the article are used by the authors to confirm the theoretical results. We have also cited references that were used, and we gave the DOI in the references.

\section{Conflicts of Interest}

The authors declare that they have no conflicts of interest.

\section{Acknowledgments}

The authors are very much thankful to the reviewers for their valuable suggestions and comments that lead to improve the quality of this manuscript.

\section{References}

[1] A. J. Lotka, "Elements of physical biology," Nature, vol. 116, no. $2917,1925$.

[2] V. Volterra, "Variazioni e fluttuazioni del numero di individui in specie animali conviventi," Mem. R. Acad. Lincei, vol. 2, pp. 31-113, 1926.

[3] B. S. Goh, "Global stability in two species interactions," Journal of Mathematical Biology, vol. 3, no. 3-4, pp. 313-318, 1976.

[4] S. B. Hsu, T. W. Hwang, and Y. Kuang, "Global analysis of the Michaelis-Menten-type ratio-dependent predator-prey system," Journal of Mathematical Biology, vol. 42, no. 6, pp. 489-506, 2001.

[5] T. W. Hwang, "Uniqueness of the limit cycle for Gause-type predator-prey systems," Journal of Mathematical Analysis and Applications, vol. 238, no. 1, pp. 179-195, 1999.

[6] Y. Kuang and E. Beretta, "Global qualitative analysis of a ratiodependent predator-prey system," Journal of Mathematical Biology, vol. 36, no. 4, pp. 389-406, 1998.

[7] S. Lenhart and J. T. Workman, Optimal Control Applied to Biological Models, CRC Press, 2007.

[8] O. K. Shalsh and S. al-Nassir, "Dynamics and optimal harvesting strategy for biological models with Beverton Holt growth," Iraqi Journal of Science, vol. 223-232, pp. 223-232, 2020.

[9] Y. Xia, J. Cao, and M. Lin, "Discrete-time analogues of predator-prey models with monotonic or nonmonotonic 
functional responses," Nonlinear Analysis Real World Applications, vol. 8, no. 4, pp. 1079-1095, 2007.

[10] A. A. Elsadany and A. E. Matouk, "Dynamical behaviors of fractional-order Lotka-Volterra predator-prey model and its discretization," Journal of Applied Mathematics and Computing, vol. 49, no. 1-2, pp. 269-283, 2015.

[11] M. el-Shahed, J. J. Nieto, A. Ahmed, and I. M. E. Abdelstar, "Fractional-order model for biocontrol of the lesser date moth in palm trees and its discretization," Advances in Difference Equations, vol. 2017, no. 1, 2017.

[12] Y. Li, Y. Q. Chen, and I. Podlubny, "Stability of fractionalorder nonlinear dynamic systems: Lyapunov direct method and generalized Mittag-Leffler stability," Computers \& Mathematcs with Applications, vol. 59, no. 5, pp. 1810-1821, 2010.

[13] H. L. Li, L. Zhang, C. Hu, Y. L. Jiang, and Z. Teng, "Dynamical analysis of a fractional-order predator-prey model incorporating a prey refuge," Journal of Applied Mathematics and Computing, vol. 54, no. 1-2, pp. 435-449, 2017.

[14] S. Al-Nassir, "Dynamic analysis of a harvested fractional-order biological system with its discretization," Chaos, Solitons \& Fractals, vol. 152, p. 111308, 2021.

[15] D. Valério and J. Sá da Costa, "Variable-order fractional derivatives and their numerical approximations," Signal Processing, vol. 91, no. 3, pp. 470-483, 2011.

[16] S. Mondal, A. Lahiri, and N. Bairagi, "Analysis of a fractional order eco-epidemiological model with prey infection and type 2 functional response," Mathematical Methods in the Applied Sciences, vol. 40, no. 18, pp. 6776-6789, 2017.

[17] E. Ahmed, A. M. A. el-Sayed, and H. A. A. el-Saka, "Equilibrium points, stability and numerical solutions of fractionalorder predator-prey and rabies models," Journal of Mathematical Analysis and Applications, vol. 325, no. 1, pp. 542-553, 2007.

[18] A. A. El-Saka, "The fractional-order SIS epidemic model with variable population size," Journal of the Egyptian Mathematical Society, vol. 22, no. 1, pp. 50-54, 2014.

[19] M. Javidi and N. Nyamoradi, "Dynamic analysis of a fractional order prey-predator interaction with harvesting," Applied Mathematical Modelling, vol. 37, no. 20-21, pp. 8946-8956, 2013.

[20] D. Mukherjee and R. Mondal, "Dynamical analysis of a fractional order prey-predator system with a reserved area," Journal of Fractional Calculus and Applications, vol. 11, no. 1, pp. 54-69, 2020.

[21] N. Supajaidee and S. Moonchai, "Stability analysis of a fractional-order two-species facultative mutualism model with harvesting," Advances in Difference Equations, vol. 2017, no. 1, 2017.

[22] M. Caputo and M. Fabrizio, "A new definition of fractional derivative without singular kernel," Progress in Fractional Differentiation and Applications, vol. 2, pp. 73-85, 2015.

[23] K. B. Oldham and J. Spanier, The Fractional Calculus Theory and Applications of Differentiation and Integration to Arbitrary Order, Academic Press, New York, NY, USA, 1974.

[24] I. Podlubny, Fractional Differential Equations, Academic Press, New York, NY, USA, 1999.

[25] L. E. S. Ramirez and C. F. M. Coimbra, "On the selection and meaning of variable order operators for dynamic modeling," International Journal of Differential Equations, vol. 2010, Article ID 846107, 16 pages, 2010.
[26] A. A. Kilbas, H. M. Srivastava, and J. J. Trujillo, Theory and Applications of Fractional Differential Equations, Elsevier, Amsterdam, Netherlands, 2006.

[27] Z. M. Odibat and N. T. Shawagfeh, "Generalized Taylor's formula," Applied Mathematics and Computation, vol. 186, no. 1, pp. 286-293, 2007.

[28] K. Diethelm, The Analysis of Fractional Differential Equations, Springer, Berlin, Germany, 2010.

[29] K. S. Miller and B. Ross, An Introduction to the Fractional Calculus and Fractional Differential Equations, Wiley, New York, NY, USA, 1993.

[30] E. Ahmed, A. M. A. El-Sayed, and H. A. A. El-Saka, "On some Routh-Hurwitz conditions for fractional order differential equations and their applications in Lorenz, Rossler, Chua and Chen systems," Physics Letters, Section A: General, Atomic and Solid State Physics, vol. 358, no. 1, pp. 1-4, 2006. 